\title{
Commentary The severity of sepsis: yet another factor influencing glycemic control
}

James S Krinsley

Stamford Hospital, 190 West Broad Street, Stamford, CT 06902, USA

Corresponding author: James S Krinsley, jkrinsley@stamhealth.org

Published: 25 November 2008

Critical Care 2008, 12:194 (doi:10.1186/cc7111)

This article is online at http://ccforum.com/content/12/6/194

(C) 2008 BioMed Central Ltd

See related research by Waeschle et al., http://ccforum.com/content/12/5/R129

\begin{abstract}
The present commentary provides a brief overview of the evolving literature on glycemic management in critically ill patients. Recent interventional studies have been plagued by high rates of severe hypoglycemia among patients, particularly those with sepsis. The investigation by Waeschle and colleagues adds to our knowledge about the relationship between the severity of sepsis and glycemic dysregulation. The severity of sepsis is shown to correlate with the risk of sustaining hyperglycemia as well as critical hypoglycemia.
\end{abstract}

The previous issue of Critical Care featured work by Waeschle and coinvestigators describing the interaction between two topics of great interest to intensivists: sepsis and glycemic management [1]. The publication of the first Leuven trial ushered into intensive care a new treatment paradigm: monitoring and treating even modest degrees of hyperglycemia in critically ill patients improved outcomes [2]. We have learned a great deal in the 7 years since this landmark study.

Van den Berghe and colleagues treated a narrow population of surgical intensive care unit (ICU) patients: all were mechanically ventilated, and $63 \%$ had undergone cardiovascular surgery. The rate of severe hypoglycemia $(\mathrm{SH})$ (blood glucose $<40 \mathrm{mg} / \mathrm{dl}$ ) was low, perhaps in part due to the participation of study physicians not directly involved in patient care who actively monitored the glycemic management protocol [2]. Confirmation of the benefit of intensive glycemic monitoring and treatment followed 3 years later in the Stamford trial, a before and after intervention completed in a mixed medicalsurgical ICU setting [3]. Tellingly, there was no difference in the rate of $\mathrm{SH}$ between the two groups in that study.

Subsequent trials met a somewhat different fate. The second Leuven trial, performed in a medical ICU, demonstrated decreased mortality in the predefined population of patients treated for more than 3 days, but not in the entire treated population [4]. The number of patients sustaining $\mathrm{SH}$ was strikingly higher in the treated group compared with that seen in the control arm (18.7\% versus $3.1 \%$, respectively). Two studies initially reported in 2006 - the Efficacy of Volume Substitution and Insulin Therapy in Severe Sepsis (VISEP) trial, a multicenter investigation limited to patients with severe sepsis; and the Glucontrol trial, a multicenter study in a mixed group of medical and surgical ICUs - were terminated prematurely by their respective safety boards, in part due to excessive rates of $\mathrm{SH}$ in the treatment arms $[5,6]$.

More recently, two studies in large observational cohorts have identified sepsis as an important risk factor for the development of $\mathrm{SH}[7,8]$. One group found that a single episode of $\mathrm{SH}$ was associated with a significant increased risk of mortality, using multivariable regression analysis as well as a case-control methodology [8].

Two important conclusions emerge from this body of research. It matters how glycemic control is implemented: $\mathrm{SH}$ is deleterious. Moreover, this issue is especially relevant in patients with sepsis, who appear to be at heightened risk for the development of $\mathrm{SH}$.

It is in this context that the study by Waeschle and coworkers makes a valuable contribution to the literature on glycemic management of critically ill patients [1]. The investigators analyzed various indices of glycemic control in a cohort of 191 patients, treated with an insulin protocol targeting 80 to $140 \mathrm{mg} / \mathrm{dl}$, who were admitted with sepsis or who developed sepsis during admission to a predominantly surgical ICU. Patients were stratified by the severity of sepsis: sepsis patients $(n=47)$, severe sepsis patients $(n=83)$ and septic shock patients $(n=61)$. 
The percentage of patients with at least one episode of $\mathrm{SH}$ increased as the severity of the category of sepsis increased: $2.1 \%, 6.0 \%$ and $11.5 \%$ for patients with sepsis, severe sepsis and septic shock, respectively. Unfortunately the authors do not indicate the relative percentage of insulinrelated or spontaneously occurring critical hypoglycemia. In the study by Krinsley and Grover, $27.5 \%$ of the patients with $\mathrm{SH}$ had not received any insulin within 12 hours of the occurrence of hypoglycemia [8].

The severity of sepsis also impacted the occurrence of hyperglycemia; the percentage of patients with blood glucose $>179 \mathrm{mg} / \mathrm{dl}$ was $55.3 \%, 73.5 \%$ and $88.5 \%$ for patients with sepsis, severe sepsis and septic shock, respectively. Moreover, both the duration and intensity of insulin therapy was positively associated with the severity of sepsis. The median duration of continuous insulin therapy was 83.4 hours, 101.1 hours and 217.6 hours, and the median insulin dose per day applied over the entire ICU stay was 33.2 units, 36.3 units and 45.0 units for patients with sepsis, severe sepsis and septic shock, respectively. These data reflect the increased degree of metabolic derangement associated with full-blown septic shock.

Interestingly, the study by Waeschle and colleagues also evaluated glycemic variability, defined as the standard deviation (SD) of the mean glucose level occurring during the sepsis episodes. Multivariable regression analysis demonstrated that the SD was positively associated with the development of $\mathrm{SH}$. Moreover, increased glycemic variability was strongly associated with mortality in this cohort: $24.0 \%$ versus $2.5 \%$, comparing patients with $\mathrm{SD}>20 \mathrm{mg} / \mathrm{dl}$ or $<20 \mathrm{mg} / \mathrm{dl}$. Indeed, every patient with severe sepsis and septic shock who died had a SD $>20 \mathrm{mg} / \mathrm{dl}$ [1]. These results corroborate recent investigations in medical-surgical ICU populations $[9,10]$, in a single-center surgical ICU cohort [11] and in a cohort of patients specifically with sepsis [12] that demonstrated the significant effect of glycemic variability on outcome of critically ill patients.

How do we integrate the findings of the current study into clinical practice? Sepsis is clearly associated with disturbances in glucose regulation, and the occurrence of hypoglycemia (both spontaneous and treatment-related) and hyperglycemia increase along the continuum from sepsis through severe sepsis to septic shock. Glycemic variability may also be positively associated with the severity of sepsis, although the current study was underpowered to demonstrate this unequivocally. The failure of the VISEP investigators to complete enrollment for their trial of intensive insulin therapy in a population of septic shock, due to the very high rate of $\mathrm{SH}$, is quite understandable in this context.

Safe, effective glycemic control may be a useful paradigm to replace tight glycemic control, especially in a population such as the patients described in the current investigation - that is inherently susceptible to major disturbances in glycemic regulation [13]. The principles of this emerging model include the choice of a glycemic target that does not yield excessive rates of severe hypoglycemia and the optimal implementation of protocols, assisted by the possible use of data outcome tools, electronic algorithms and, eventually, continuous or near-continuous glucose monitors. Successful application of this approach offers the best opportunity for clinicians to maintain blood glucose levels in the chosen range, to minimize glycemic variability and to yield the best outcomes for our patients.

\section{Competing interests}

Roche Diagnostics Corporation: Speakers Bureau; Consulting OptiScan Biomedical: Research funding.

\section{References}

1. Waeschle RM, Moerer O, Hilgers R, Herrmann P, Neumann $P$, Quintel M: The impact of the severity of sepsis on the risk of hypoglycemia and glycemic variability. Crit Care 2008, 12:R129.

2. Van den Berghe G, Wouters P, Weekers F, Verwaest C, Bruyninckx F, Schetz M, Vlasselaers D, Ferdinande P, Lauwers P, Bouillon R: Intensive insulin therapy in the critically ill patients. N Engl J Med 2001, 345:1359-1367.

3. Krinsley JS: Effect of an intensive glucose management protocol on the mortality of critically ill patients. Mayo Clin Proc 2004, 79:992-1000

4. Van den Berghe G, Wilmer A, Hermans G, Meersseman W, Wouters PJ, Milants I, Van Wijngaerden E, Bobbaerts H, Bouillon $\mathrm{R}$ : Intensive insulin therapy in the medical ICU. $N$ Engl J Med 2006, 354:449-461.

5. Brunkhorst FM, Engel C, Bloos F, Meier-Hellmann A, Ragaller M, Weiler N, Moerer O, Gruendling M, Oppert M, Grond S, Olthoff D, Jaschinski U, John S, Rossaint R, Welte T, Schaefer M, Kern P, Kuhnt E, Kiehntopf M, Hartog C, Natanson C, Loeffler M, Reinhart K, German Competence Network Sepsis (SepNet): Intensive insulin therapy and pentastarch resuscitation in severe sepsis. N Engl J Med 2008, 358:125-139.

6. Devos P, Preiser JC, Mélot C, on behalf of the Glucontrol Steering Committee: Impact of tight glucose control by intensive insulin therapy on ICU mortality and the rate of hypoglycaemia: final results of the Glucontrol study [abstract]. Intensive Care Med 2007, 33:S189.

7. Vriesendorp TM, DeVries JH, van Santen S, Moeniralam HS, de Jonge E, Roos YB, Schultz MJ, Rosendaal FR, Hoekstra JB: Evaluation of short-term consequences of hypoglycemia in an intensive care unit. Crit Care Med 2006, 34:2714-2718.

8. Krinsley JS, Grover A: Severe hypoglycemia in critically ill patients: risk factors and outcomes. Crit Care Med 2007, 35: 2262-2267.

9. Egi M, Bellomo R, Stachowski E, French CJ, Hart G: Variability of blood glucose concentration and short-term mortality in critically ill patients. Anesthesiology 2006, 105:244-252.

10. Krinsley JS: Glycemic variability: a strong independent predictor of mortality in critically ill patients. Crit Care Med 2008, 36: 3008-3013.

11. Dossett LA, Cao H, Mowery NT, Dortch MJ, Morris JM Jr, May AK: Blood glucose variability is associated with mortality in the surgical intensive care unit. Am Surg 2008, 74:679-685.

12. Ali NA, O'Brien JM Jr, Dungan K, Phillips GS, Marsh CB, Lemeshow S, Connors AF, Prieser JC: Glucose variability and mortality in patients with sepsis. Crit Care Med 2008, 36: 2316-2321.

13. Krinsley JS, Preiser JC: Moving beyond tight glycemic control to safe, effective glycemic control. Crit Care 2008, 12:149-151. 